\title{
Reverse propagation of femtosecond pulses in optical fibers
}

\author{
Mankei Tsang and Demetri Psaltis \\ Department of Electrical Engineering, California Institute of Technology, Pasadena, California 91125
}

Fiorenzo G. Omenetto

Physics Division, P-23, MS H803, Los Alamos National Laboratory, Los Alamos, New Mexico 87545

Received March 3, 2003

\begin{abstract}
We present a numerical technique for reversing femtosecond pulse propagation in an optical fiber, such that given any output pulse it is possible to obtain the input pulse shape by numerically undoing all dispersion and nonlinear effects. The technique is tested against experimental results, and it is shown that it can be used for fiber output pulse optimization in both the anomalous and normal dispersion regimes. (C) 2003 Optical Society of America

OCIS codes: $\quad 060.4370,060.5530,060.7140,190.4370,190.5530,190.7110$.
\end{abstract}

Dispersion and nonlinear effects have been the bottleneck of ultrafast pulse propagation in an optical fiber. Various schemes, for example, optical solitons ${ }^{1}$ and optical phase conjugation (OPC), ${ }^{2,3}$ have been proposed to compensate for these effects, yet the high-order distortions including third-order dispersion (TOD), self-steepening, and Raman scattering remain to be defeated. Femtosecond power delivery in a normally dispersive fiber, which is useful for biomedical applications, is especially difficult to achieve because normal dispersion and nonlinear effects always tend to broaden and distort a pulse. Another scheme is to embrace all the effects and adopt an adaptive optimization method, typically in the form of genetic algorithm, hoping that modulating the input pulse shape can produce an output with desirable properties. ${ }^{4-6}$ Although an adaptive method can compensate for pulse propagation distortions and unknown experimental variables, it does not make full use of our theoretical knowledge of optical fiber ultrafast pulse propagation and may therefore be time consuming and suboptimal.

In this Letter we show that by reversing the nonlinear pulse propagation equation it is possible to theoretically predict the exact input pulse shape that gives a desired output of a fiber. All dispersion and nonlinear effects can be incorporated into the simulation, and hence one can produce any kind of pulse shape at the output end by shaping the input pulse appropriately according to the calculated result. In conjunction with currently available femtosecond pulse-shaping techniques such as $4 f$ pulse shaping, ${ }^{7}$ this approach is especially suited to provide custom-shaped high-power ultrafast pulse delivery both in normally and anomalously dispersive fibers.

The nonlinear pulse propagation equation in a fiber is given by the general form ${ }^{1}$

$$
\frac{\partial A(z, T)}{\partial z}=\{\hat{D}+\hat{N}[A(z, T)]\} A(z, T),
$$

where $A(z, T)$ is the pulse envelope, $\hat{D}$ is the linear operator that includes absorption and all dispersion effects, and $\hat{N}$ is the nonlinear operator that includes all nonlinear effects and is a function of $A(z, T)$. Mathematically, the output is obtained by application of the propagation operator to the input:

$$
A(L, T)=\exp \left\{L \hat{D}+\int_{0}^{L} \hat{N}[A(z, T)] \mathrm{d} z\right\} A(0, T),
$$

where $L$ is the length of the fiber. The input can also be expressed in terms of the output by application of the reverse propagation operator:

$$
A(0, T)=\exp \left\{-L \hat{D}-\int_{0}^{L} \hat{N}[A(z, T)] \mathrm{d} z\right\} A(L, T) .
$$

To solve this equation and derive the input pulse shape given the output, we use the standard Fourier split-step method:

$A(z, T) \approx \exp (-h \hat{D}) \exp \{-h \hat{N}[A(z+h, T)]\} A(z+h, T)$,

in each step of which the linear and nonlinear effects on a pulse shape are evaluated separately for a small propagation distance $h$.

As a comparison, let us consider the OPC technique in the operator notation. Figure 1 depicts schematically the OPC method and the reverse propagation method. By conjugating Eq. (2) and comparing the result with Eq. (3), one can see that OPC can reconstruct an input pulse if $\hat{D}$ and $\hat{N}$ contain only operators with the property $\hat{M}=-\hat{M}^{*}$. This restriction precludes many important phenomena, such as loss, TOD, and
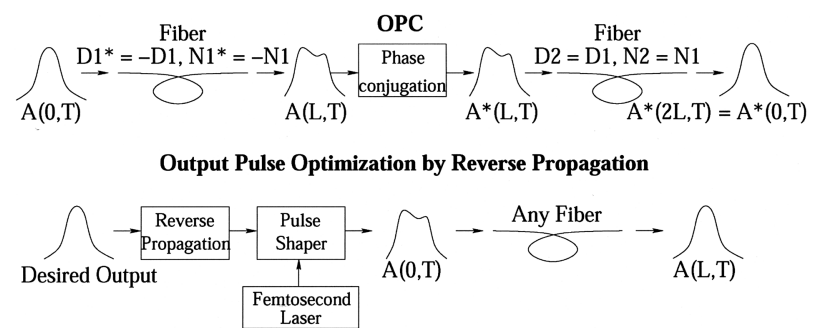

Fig. 1. Comparison of OPC and reverse propagation. 
self-steepening, that may severely hamper the accuracy of pulse reconstruction by OPC, especially in the femtosecond regime. In a numerical simulation of reverse propagation, however, there is no such limitation, and all effects can be included to yield the optimal input pulse shape.

To model femtosecond pulse propagation, we choose the linear operator $\hat{D}$ to be

$$
\hat{D}=-\frac{\alpha}{2}-j \frac{\beta_{2}}{2} \frac{\partial^{2}}{\partial T^{2}}+\frac{\beta_{3}}{6} \frac{\partial^{3}}{\partial T^{3}} .
$$

The first term corresponds to loss, the second term corresponds to group-velocity dispersion (GVD), and the third term corresponds to TOD. The higher-order dispersion terms are neglected because of the relatively short length considered here but can be easily included if the need arises.

The nonlinear operator $\hat{N}$ is

$$
\hat{N}=j \gamma\left[|A|^{2}+\frac{j}{\omega_{0}} \frac{1}{A} \frac{\partial}{\partial T}\left(|A|^{2} A\right)-T_{R} \frac{\partial|A|^{2}}{\partial T}\right]
$$

The first term corresponds to optical Kerr effect, the second term corresponds to self-steepening, and the third term corresponds to intrapulse Raman scattering.

To test the validity of reverse propagation, in an experiment we launch a 150 -fs positively chirped hyperbolic secant pulse with a peak power of $1 \mathrm{~kW}$ in a 10-m-long Corning SMF-28 fiber. ${ }^{6}$ From frequency-resolved optical gating measurements we obtain the amplitude and phase of both input and output pulses. The output pulse is then reverse propagated in a computer simulation as in Fig. 2. The output pulse shape is plotted at $z=0 \mathrm{~m}$ at the top of the graph, and propagation effects are reversed numerically as the pulse goes from $z=0 \mathrm{~m}$ to $z=-10 \mathrm{~m}$. The simulated input from reverse propagation is compared with the experimental input in Fig. 3. Both pulses are remarkably similar, with nearly identical amplitudes and positive chirp, showing that the reverse propagation theory is consistent with experimental results.

Reverse propagation can be used to calculate the proper input pulse shape that provides a narrow pulse at the output. As a numerical example, let us consider a 100-m-long conventional dispersion-shifted fiber with parameters $\beta_{2}=-1 \mathrm{ps}^{2} / \mathrm{km}$, $\beta_{3}=0.1 \mathrm{ps}^{3} / \mathrm{km}, \gamma=1.5 \mathrm{~W}^{-1} \mathrm{~km}^{-1}$, and $T_{R}=3 \mathrm{fs}$ at $\lambda_{0}=1550 \mathrm{~nm}$. A fundamental soliton with a pulse width $T_{0}$ of $100 \mathrm{fs}$ can propagate in this fiber with a peak power of $67 \mathrm{~W}$ but will experience distortions as a result of TOD, self-steepening, and soliton self-frequency shift. To obtain better output, we reverse the propagation of a desirable pulse shape, say a chirped sech pulse with a pulse profile $A(0, T)=P_{0}^{1 / 2} \operatorname{sech}\left(T / T_{0}\right) \exp \left[-i C\left(T / T_{0}\right)^{2} / 2\right]$, peak power $P_{0}=67 \mathrm{~W}$, and $\operatorname{chirp} C=1$.

Figure 4 shows the reverse propagation of the desired output along the fiber. The peak intensity of the optimized input pulse during propagation is lower than that of a fundamental soliton, thereby avoiding the high-order nonlinear distortions. To obtain an even

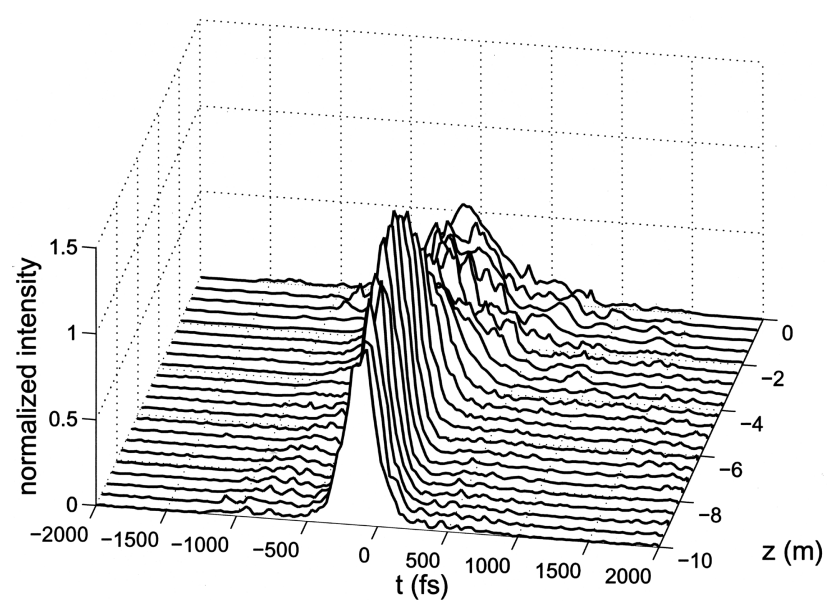

Fig. 2. Reverse propagation of an experimental output pulse. The experimental output pulse shape is plotted at $z=0 \mathrm{~m}$ and numerically propagates in reverse from $z=0 \mathrm{~m}$ to $z=-10 \mathrm{~m}$.
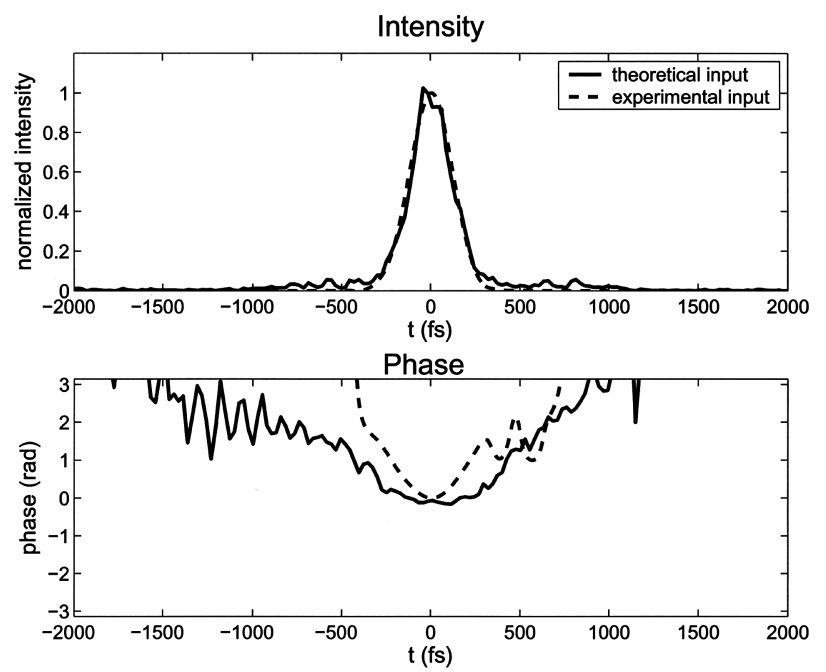

Fig. 3. Comparison of the input obtained from reverse propagation and the actual experimental input.

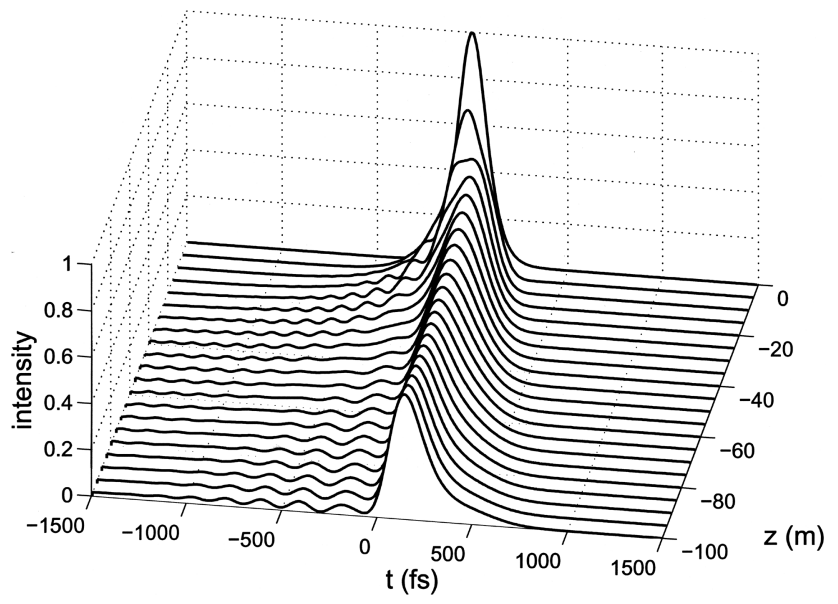

Fig. 4. Reverse propagation of a chirped sech pulse at $\lambda_{0}=1550 \mathrm{~nm}$. 

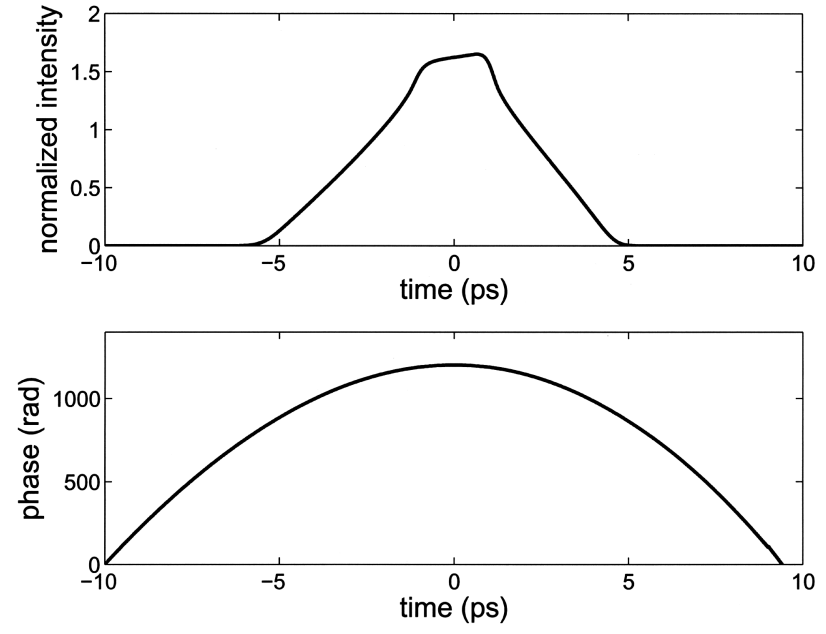

Fig. 5. Amplitude and phase of the optimal input that produces the desired sech output pulse shape at $\lambda_{0}=800 \mathrm{~nm}$.

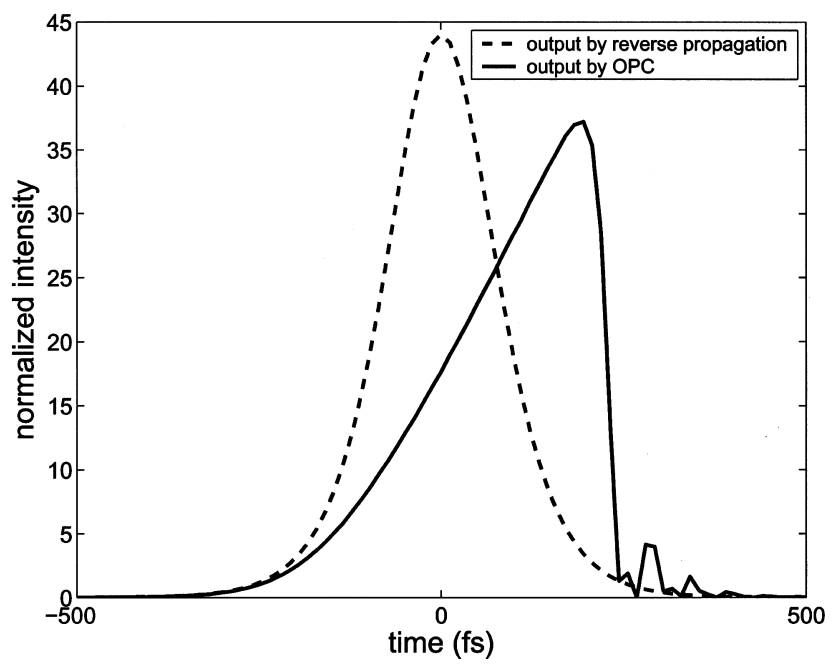

Fig. 6. Compared with the ideal output pulse shape produced by reverse propagation and pulse shaping, the OPC output is significantly distorted by high-order effects.

shorter pulse, we would need to include higher-order linear and nonlinear terms in Eqs. (5) and (6).

It must be stressed that the reverse propagation method is not limited to near-soliton conditions but can be applied to any fiber in both dispersion regimes. For our second numerical example consider a 1-m-long normally dispersive single-mode fiber at $\lambda_{0}=800 \mathrm{~nm}$, with parameters $\beta_{2}=40 \mathrm{ps}^{2} / \mathrm{km}, \beta_{3}=0.03 \mathrm{ps}^{3} / \mathrm{km}$, and $\gamma=9 \mathrm{~W}^{-1} \mathrm{~km}^{-1}$. $\quad$ A $100-\mathrm{fs}$ unchirped hyperbolic secant pulse, centered at $800 \mathrm{~nm}$, with peak power $P_{0}=20 \mathrm{~kW}$ and total energy $E=4 \mathrm{~nJ}$ is given as our desired output. Figure 5 shows the optimal input pulse amplitude and phase. Qualitatively speaking the pulse shape is asymmetric to compensate for TOD, self-steepening, and Raman scattering, and the negative chirp $(C=-0.1$ by polynomial fitting) focuses the pulse by GVD. In an experiment the large chirp can be imposed by a grating pair before $4 f$ pulse shaping.

We also investigate numerically the $\mathrm{OPC}$ technique, as depicted in Fig. 1, using the same criteria as those above as a comparison. Figure 6 shows the resultant output pulse by OPC compared with the ideal output pulse shape that can be produced by reverse propagation and pulse shaping. The OPC output pulse is distorted by high-order effects, whereas reverse propagation and pulse shaping, taking all the high-order effects into account, produce a better output.

In summary, we have shown in this Letter that reverse propagation is a feasible method of predicting the optimal input pulse shape that will yield any desired output in a well-characterized fiber, defeating all high-order dispersive and nonlinear effects that may be detrimental to other output optimization schemes. The theory is successfully tested against experimental results, and its application to femtosecond pulse delivery through fibers in both dispersion regimes is presented numerically. The interface of reverse propagation code to a pulse shaper can be envisaged for short propagation lengths, so that the proper modulation is applied to the input pulse by the programmable optical modulator of choice. For longer distances, practical realizations become more complex as linear distortions become too large to be overcome by the available modulators alone. In this case linear compensators can be combined with programmable modulators and reverse propagation predictions to compensate for all distortions.

This work was supported primarily by the Engineering Research Centers Program of the National Science Foundation under award EEC-9402726. M. Tsang's e-mail address is mankei@sunoptics.caltech.edu.

\section{References}

1. G. P. Agrawal, Nonlinear Fiber Optics (Academic, San Diego, Calif., 2001).

2. A. Yariv, D. Fekete, and D. M. Pepper, Opt. Lett. 4, 52 (1979).

3. R. A. Fisher, B. R. Suydam, and D. Yevick, Opt. Lett. 8, 611 (1983).

4. R. S. Judson and H. Rabitz, Phys. Rev. Lett. 68, 1500 (1992).

5. F. G. Omenetto, B. P. Luce, and A. J. Taylor, J. Opt. Soc. Am. B 16, 2005 (1999).

6. F. G. Omenetto, A. J. Taylor, M. D. Moores, and D. H. Reitze, Opt. Lett. 26, 938 (2001).

7. A. M. Weiner, J. P. Heritage, and E. M. Kirschner, J. Opt. Soc. Am. B 5, 1563 (1988).

8. S. W. Clark, F. O. Ilday, and F. W. Wise, Opt. Lett. 26, 1320 (2001). 